\title{
Über die elektromagnetische Drehnng der Polarisationsebene einiger Säuren und Salze in verschiedenen Lösungsmitteln.
}

\author{
Von \\ Otto Humburg.
}

Um für die Beurteilung der Frage nach dem Zusammenhang zwischen der elektromagnetischen Drehung der Polarisationsebene in Lösungen von Elektrolyten und dem Dissociationszustande, in welchem sich die letzteren befinden, weiteres Material zu schaffen, habe ich die nachstehend beschriebenen Versuche ausgefuhrt.

Die Messungen wurden mit demselben Apparat und nach demselben Verfahren ausgeführt, wie die des Herrn Schönrock ${ }^{1}$ ). Zur Bestimmung der Konzentration der Lösungen wurden die Flüssigkeiten und die bis zum konstanten Gewicht teils bei $100^{\circ}$, teils über Schwefelsäure im Vakuum getrockneten Salze in einem Kolben mit eingeschliffenem Glasstopfen zunächst für sich und darauf mit dem Lösungsmittel auf mg genau gewogen. Bei der Berechnung der Molekulardrehungen wurde das Perkinsche Verfahren eingeschlagen. In 100 Gewichtsteilen der Lösung sind $a$ Gewichtsteile des Körpers, dessen Rotation bestimmt werden soll, und $b$ Gewichtsteile des Lösungsmittels enthalten. Da nun das Gewicht einer bestimmten Menge Substanz gleich dem Produkt aus der Anzahl der Molekeln und dem Gewicht der einzelnen Molekel ist, so muss sein

$$
\begin{array}{rlrl}
a & =m M_{1} & b & =n M_{z} \\
m & =\frac{a}{M_{1}} & n & =\frac{b}{M_{2}} .
\end{array}
$$

Es verhält sich mithin die Anzahl der Molekeln des Salzes zu der des Lösungsmittels wie $\frac{a}{M_{1}}: \frac{b}{M_{2}}$ oder auf 1 Molekel der zu untersuchenden Substanz kommen $\frac{b M_{1}}{a M_{2}}$ Molekeln der Flüssigkeit. Das Gesamt-

1) Diese Zeitschr. 11, 753. 1893.

Zeitschrift f. physik. Chemie. XII. 
molekulargewicht der Lösung, das für die Formel

$$
d_{m}=\frac{w . M}{w_{H_{2} 0 . s .18}}
$$

erfordert wird, berechnet sich aus dem oben Entwickelten

$$
\begin{aligned}
& M=M_{1}+\frac{b M_{1}}{a M_{2}} M_{2} \\
& M=\frac{M_{1}}{a}(a+b)=\frac{M_{\mathrm{i}} \cdot 100}{a},
\end{aligned}
$$

80 dass man als Endgleichung für die molekulare Drehung der Lösung erhält

$$
d_{n}=\frac{w \cdot M_{1} \cdot 100}{w_{H_{2}} 0 \cdot s \cdot 18 \cdot a} \text {. }
$$

Durch Subtraktion der dem Lösungsmittel entsprechenden Rotation von der molekularen Drehung erhält man dann die molekulare Ablenkung der Polarisationsebene durch die gelöste Substanz.

Als Lösungsmittel für die Fettsäuren wurden Alkohol und Äther, deren Anwendung wohl am nächsten lag, aus nahe liegenden Gründen nicht gewählt. Bei Äthylalkohol lag die Gefahr einer langsamen Esterbildung sehr nahe, die die Resultate stark beeinflusst hätte, und Äther war wegen der zu grossen Flüchtigkeit ausgeschlossen, da bei allen diesen Untersuchungen die Konstanz der Mischung Grundbedingung ist und das Verdampfen kleiner Mengen Äther während der verschiedenen Manipulationen nicht $\mathrm{zu}$ vermeiden ist. Deshalb entschloss ich mich, auf den Rat von Herrn Dr. Jahn, zu Benzol und Toluol für die Fettsäuren, während für die anorganischen Salze neben Wasser der Methylalkohol gewählt wurde.

Bevor an die eigentliche Untersuchung geschritten werden konnte, musste die elektromagnetische Drehung der Polarisationsebene für das Wasser, dessen Rotation als Einheit aller ferneren Messungen zu setzen war, sowie für die drei anderen Lösungsmittel, Benzol, Toluol und $\mathrm{Me}-$ thylalkohol, bestimmt werden. Die folgenden Tabellen, in denen die direkt gefundenen und die daraus berechneten Zahlen niedergelegt sind, enthalten in der ersten Kolumne die beobachtete Drehung der Polarisationsebene in Minuten. Die Zahlen in der mit $J$ iberschriebenen Reihe geben die durch das Silbervoltameter gemessene Intensität des galvanischen Stromes in Ampère an und $w$ bedeutet die aus diesen beiden Werten für die Stromintensität gleich 1 Ampère berechnete Ablenkung der Polarisationsebene. $s \frac{16}{4}$ ist die Dichte der Lösung bei $16^{\circ}$ auf Wasser bei $4^{0}$ als Einheit bezogen, nach der Reduktion des Ge- 
Ther die elektromagnet. Drehang der Polarisationsebene einiger Sauren etc. 403 wichtes anf den luftleeren Raum. Die Zahlen unter $d_{m}$ sind endlich die gesnchten Molekulardrehungen.

Tabellle I.

\begin{tabular}{|c|c|c|c|c|c|}
\hline Bubetanx & $D$ & $J$ & 20 & $s$ & $d_{m}$ \\
\hline \multirow[t]{2}{*}{ Wasser } & \multirow[t]{2}{*}{$\begin{array}{l}65 \cdot 950 \\
66 \cdot 350 \\
65 \cdot 335 \\
65 \cdot 210 \\
73 \cdot 418\end{array}$} & \multirow[t]{2}{*}{$\begin{array}{l}0.65071 \\
0.66003 \\
0.64978 \\
0.64180 \\
0.72838\end{array}$} & $\begin{array}{l}101.350 \\
100.525 \\
100.548 \\
101.605 \\
100.796\end{array}$ & \multirow[b]{2}{*}{1} & \multirow[b]{2}{*}{1} \\
\hline & & & 100.965 & & \\
\hline \multirow[t]{2}{*}{ Benzol } & \multirow[t]{2}{*}{$\begin{array}{l}129.84 \\
159.73 \\
142.49 \\
126.25\end{array}$} & \multirow[t]{2}{*}{$\begin{array}{l}0.56872 \\
0.69701 \\
0.62612 \\
0.55575\end{array}$} & $\begin{array}{l}228 \cdot 353 \\
229 \cdot 167 \\
227 \cdot 577 \\
227 \cdot 165\end{array}$ & \multirow[b]{2}{*}{0.88422} & \multirow[b]{2}{*}{11.0629} \\
\hline & & & 228.066 & & \\
\hline \multirow[t]{2}{*}{ Toluol } & \multirow[t]{2}{*}{$\begin{array}{l}121.64 \\
118.68 \\
121.15 \\
119.20\end{array}$} & \multirow[t]{2}{*}{$\begin{array}{l}0.58686 \\
0.57603 \\
0.59155 \\
0.57826\end{array}$} & $\begin{array}{l}207.273 \\
206.034 \\
204.809 \\
206.133\end{array}$ & \multirow[b]{2}{*}{0.8694} & \multirow[b]{2}{*}{12.0256} \\
\hline & & & 206.062 & & \\
\hline \multirow[t]{2}{*}{ Methylalkohol } & \multirow[t]{2}{*}{$\begin{array}{l}49.721 \\
49.486 \\
49.633 \\
49.847\end{array}$} & \multirow[t]{2}{*}{$\begin{array}{l}0.69601 \\
0.69209 \\
0.69094 \\
0.69024\end{array}$} & $\begin{array}{l}71.437 \\
71.502 \\
71.834 \\
\mathbf{7 2 . 2 1 7} \\
\end{array}$ & \multirow[b]{2}{*}{0.7941} & \multirow[b]{2}{*}{1.5898} \\
\hline & & & 71.7475 & & \\
\hline
\end{tabular}

Der von C. A. F. Kahlbaum bezogene Eisessig wurde durch Ausfrieren von den letzten Spuren aufgelösten Wassers befreit und darauf die elektromagnetische Drehung der reinen Säure bestimmt.

Tabelle II.

\begin{tabular}{l||l|l|l|l|l}
\hline Essigsaure & 54.108 & 0.68619 & 78.852 & & \\
& 53.401 & 0.68724 & 77.703 & & \\
& 28.517 & 0.35465 & 80.409 & & \\
& 28.847 & 0.36583 & 78.854 & & \\
& & & 78.955 & 1.0557 & 2.4746
\end{tabular}

Durch Mischen genau gewogener Mengen reiner Säure und Wasser ergaben sich folgende vier Lösungen, deren Ablenkung der Polarisationsebene ermittelt wurde.

Tabelle III.

\begin{tabular}{c||c|c|c|c|c}
\hline Essigsañe & 65.146 & 0.67800 & 96.085 & & \\
in Wasser & 69.266 & 0.73196 & 94.631 & & 7.6682 \\
$a=39.077 \%$ & 69.309 & 0.73136 & $\mathbf{9 4 . 7 6 7}$ & & $\mathbf{5 . 2 0 8 4}$ \\
\hline
\end{tabular}




\begin{tabular}{|c|c|c|c|c|c|}
\hline Bubstanz & $D$ & $J$ & $w$ & 8 & $d_{m}$ \\
\hline \multirow[t]{2}{*}{$a=18.204 \%$} & \multirow[t]{2}{*}{$\begin{array}{l}71.890 \\
75.594 \\
74.315\end{array}$} & \multirow[t]{2}{*}{$\begin{array}{l}0.73286 \\
0.76416 \\
0.75492\end{array}$} & $\begin{array}{l}98.096 \\
98.925 \\
98.441\end{array}$ & \multirow[b]{2}{*}{1.0251} & $\begin{array}{l}17.462 \\
15.011\end{array}$ \\
\hline & & & 98.487 & & 2.451 \\
\hline \multirow[t]{2}{*}{$\begin{array}{l}\text { III. } \\
a=12.780 \%\end{array}$} & \multirow[t]{2}{*}{$\begin{array}{l}77 \cdot 117 \\
77 \cdot 144 \\
\mathbf{7 5 \cdot 8 6 5}\end{array}$} & \multirow[t]{2}{*}{$\begin{array}{l}0.78205 \\
0.77952 \\
0.76133\end{array}$} & $\begin{array}{l}98 \cdot 609 \\
98.964 \\
99 \cdot 647\end{array}$ & \multirow[b]{2}{*}{1.0177} & $\begin{array}{l}25 \cdot 205 \\
22.800\end{array}$ \\
\hline & & & 99.073 & & 2.405 \\
\hline \multirow[t]{2}{*}{$\begin{array}{c}\text { IV. } \\
a=7.766 \%\end{array}$} & \multirow[t]{2}{*}{$\begin{array}{l}69.654 \\
70.994 \\
62.581\end{array}$} & \multirow[t]{2}{*}{$\begin{array}{l}0.68560 \\
0.70334 \\
0.62328\end{array}$} & $\begin{array}{l}101 \cdot 597 \\
100 \cdot 938 \\
100 \cdot 404\end{array}$ & \multirow[b]{2}{*}{1.0109} & $\begin{array}{l}42.560 \\
40.073\end{array}$ \\
\hline & & & 100.980 & & 2.487 \\
\hline
\end{tabular}

Die aus dem Drehungsvermögen der Lösungen unter der Voraussetzung der einfachen Summation berechnete Rotation der Essigsäure differiert nur unbedeutend von dem für die reine Säure gefundenen Wert, und die mässigen Abweichungen sind durch die nicht auszuschliessenden Beobachtungsfehler bedingt. Es wurden nun durch Mischen gewogener Mengen Eisessig und Benzol bez. Toluol Lösungen von bekannter Konzentration bereitet und auf ihre Drehung untersucht. Die in Tabelle IV zusammengestellten Zahlen beweisen, dass sich auch hier die molekulare Drehung der Essigsäure nicht geändert hat.

Tabelle IV.

\begin{tabular}{|c|c|c|c|c|c|}
\hline \multirow{2}{*}{$\begin{array}{l}\text { Essigsåure } \\
\text { in Benzol } \\
a=31.317 \%\end{array}$} & \multirow[t]{2}{*}{$\begin{array}{l}130.89 \\
134.19 \\
133.82\end{array}$} & \multirow[t]{2}{*}{$\begin{array}{l}0.70751 \\
0.72749 \\
0.72287\end{array}$} & $\begin{array}{l}184.993 \\
184.456 \\
185.124\end{array}$ & \multirow[b]{2}{*}{0.9221} & $\begin{array}{l}21.1820 \\
18.6642\end{array}$ \\
\hline & & & 184.858 & & 2.5178 \\
\hline \multirow[t]{2}{*}{$\begin{array}{c}\text { II. } \\
\alpha=19.823 \%\end{array}$} & \multirow[t]{2}{*}{$\begin{array}{l}142.22 \\
139.68 \\
137.04\end{array}$} & \multirow[t]{2}{*}{$\begin{array}{l}0.70856 \\
0.69678 \\
0.69574\end{array}$} & $\begin{array}{l}200.717 \\
200.470 \\
200.632\end{array}$ & \multirow[b]{2}{*}{0.9062} & $\begin{array}{l}36.952 \\
34.435\end{array}$ \\
\hline & & & 200.606 & & 2.517 \\
\hline \multirow[t]{2}{*}{$a=10.7985 \%$} & \multirow[t]{2}{*}{$\begin{array}{l}116.19 \\
101.06 \\
103.99\end{array}$} & \multirow[t]{2}{*}{$\begin{array}{l}0.54458 \\
0.47809 \\
0.48904\end{array}$} & $\begin{array}{l}213 \cdot 353 \\
211 \cdot 387 \\
212.678\end{array}$ & \multirow[b]{2}{*}{0.8951} & $\begin{array}{l}72.7333 \\
70.2645\end{array}$ \\
\hline & & & 212.473 & & 2.4688 \\
\hline \multirow[t]{2}{*}{$\begin{array}{l}\begin{array}{l}\text { Essigsăure } \\
\text { in Toluol } \\
a==27.446 \%\end{array}\end{array}$} & \multirow[t]{2}{*}{$\begin{array}{l}134.48 \\
127.99 \\
113.81\end{array}$} & \multirow[t]{2}{*}{$\begin{array}{l}0.77460 \\
0.76968 \\
0.65690\end{array}$} & $\begin{array}{l}173.611 \\
174.128 \\
173.247\end{array}$ & \multirow[b]{2}{*}{0.9072} & $\begin{array}{l}23.0785 \\
20.7322\end{array}$ \\
\hline & & & 173.662 & & $2 \cdot 3463$ \\
\hline \multirow[t]{2}{*}{$\begin{array}{c}\text { II. } \\
a=38 \cdot 492 \%\end{array}$} & \multirow[t]{2}{*}{$\begin{array}{c}116 \cdot 25 \\
112 \cdot 94 \\
92 \cdot 178\end{array}$} & \multirow[t]{2}{*}{$\begin{array}{l}0.72085 \\
0.70140 \\
0.57021\end{array}$} & $\begin{array}{l}161.277 \\
161.014 \\
161.655\end{array}$ & \multirow[b]{2}{*}{0.9248} & $\begin{array}{l}14.9950 \\
12.5620\end{array}$ \\
\hline & & & 161.315 & & 2.4330 \\
\hline
\end{tabular}


tber die elektromagnet. Drehang der Polarisationsebene einiger Sauren etc. 405

\begin{tabular}{c||c|c|c|c|c}
\hline \hline Bubatens & $D$ & $J$ & $w$ & $s$ & $d_{m}$ \\
\hline III. & 146.14 & 0.74806 & 195.361 & & \\
$a=9.6055 \%$ & 128.17 & 0.65690 & 195.113 & & 76.2571 \\
& & 0.64182 & $\frac{194.829}{195.101}$ & \multirow{2}{*}{0.8813} & $\frac{73.8051}{2.4520}$
\end{tabular}

In gleicher Weise verfuhr man bei der Untersuchung der Propion-, Butter-, Mono- und Dichloressigsäure. Auch hier ergaben sich für die Lösungen Zahlen, die die Richtigkeit des Verdetschen Satzes und die Unabhängigkeit der molekularen Drehung von der chemischen Natur des Lösungsmittels erwiesen.

Tabelle $\mathbf{V}$.

\begin{tabular}{|c|c|c|c|c|c|}
\hline Bubstanz & $D$ & $J$ & $w$ & 8 & $d_{m}$ \\
\hline \multirow[t]{2}{*}{ Propionsæure } & \multirow[t]{2}{*}{$\begin{array}{l}55.768 \\
56.003 \\
59.011\end{array}$} & \multirow[t]{2}{*}{$\begin{array}{l}0.65580 \\
0.66562 \\
0.68141\end{array}$} & $\begin{array}{l}85.039 \\
84.137 \\
86.204\end{array}$ & \multirow[b]{2}{*}{0.9973} & \multirow[b]{2}{*}{3.4833} \\
\hline & & & $85 \cdot 127$ & & \\
\hline \multirow{2}{*}{$\begin{array}{c}\text { Propionsaure } \\
\text { in Wasser } \\
a=35.5575 \%\end{array}$} & \multirow[t]{2}{*}{$\begin{array}{l}\mathbf{5 8 . 8 6 0} \\
\mathbf{5 8 . 9 5 7} \\
\mathbf{5 8 . 1 5 8}\end{array}$} & \multirow[t]{2}{*}{$\begin{array}{l}0.60601 \\
0.59810 \\
0.59243\end{array}$} & $\begin{array}{l}\mathbf{9 6 . 9 9 9} \\
\mathbf{9 8 . 5 8 4} \\
\mathbf{9 8 . 1 6 9} \\
\end{array}$ & \multirow[b]{2}{*}{1.0253} & $\begin{array}{r}10.9602 \\
7.4673 \\
\end{array}$ \\
\hline & & & 97.917 & & 3.4929 \\
\hline \multirow[t]{2}{*}{$\begin{array}{l}\text { II. } \\
a=10.332 \%\end{array}$} & \multirow[t]{2}{*}{$\begin{array}{l}65.669 \\
60.936 \\
61.825\end{array}$} & \multirow[t]{2}{*}{$\begin{array}{l}0.65469 \\
0.60972 \\
0.61747\end{array}$} & $\begin{array}{r}100.306 \\
99.941 \\
100.126 \\
\end{array}$ & \multirow[b]{2}{*}{1.0085} & $\begin{array}{l}\mathbf{3 9 . 2 1 4} \\
\mathbf{3 5 . 7 5 9} \\
\end{array}$ \\
\hline & & & 100.124 & & 3.455 \\
\hline \multirow[t]{2}{*}{$\begin{array}{r}\text { Propionsaure } \\
\text { in Benzol } \\
a=15.0319 \%\end{array}$} & \multirow[t]{2}{*}{$\begin{array}{l}162.53 \\
164.91 \\
162.12 \\
159.95\end{array}$} & \multirow[t]{2}{*}{$\begin{array}{l}0.78011 \\
0.79439 \\
0.77937 \\
0.77152\end{array}$} & $\begin{array}{l}208.345 \\
207.592 \\
208.017 \\
207.322\end{array}$ & \multirow[b]{2}{*}{0.8958} & $\begin{array}{l}62.7980 \\
59.3263\end{array}$ \\
\hline & & & 207.819 & & 3.4717 \\
\hline \multirow[t]{2}{*}{$\begin{array}{r}\text { Propionsăure } \\
\text { in Toluol } \\
a=29.9668 \%\end{array}$} & \multirow[t]{2}{*}{$\begin{array}{l}130.01 \\
131.66 \\
129.95 \\
127.53\end{array}$} & \multirow[t]{2}{*}{$\begin{array}{l}0.74496 \\
0.76079 \\
0.75251 \\
0.74009\end{array}$} & $\begin{array}{l}174 \cdot 521 \\
173 \cdot 062 \\
172 \cdot 683 \\
172 \cdot 309 \\
\end{array}$ & \multirow[b]{2}{*}{0.9020} & $\begin{array}{l}26.0660 \\
22.6056 \\
\end{array}$ \\
\hline & & & $173 \cdot 144$ & & 3.4604 \\
\hline \multirow[t]{2}{*}{$\stackrel{\text { II. }}{a=15.8894 \%}$} & \multirow[t]{2}{*}{$\begin{array}{l}109.69 \\
108.01 \\
109 \cdot 11 \\
109.42\end{array}$} & \multirow[t]{2}{*}{$\begin{array}{l}0.57862 \\
0.57692 \\
0.57527 \\
0.57542\end{array}$} & $\begin{array}{l}189 \cdot 567 \\
187.213 \\
189.661 \\
190 \cdot 156 \\
\end{array}$ & \multirow[b]{2}{*}{0.8856} & $\begin{array}{r}54.7001 \\
\mathbf{5 1 . 2 0 3 1} \\
\end{array}$ \\
\hline & & & 189.149 & & 3.4970 \\
\hline \multirow[t]{2}{*}{$\begin{array}{c}\text { Propionsange } \\
\text { in Benzol } \\
\text { II. } a==37.2596 \%\end{array}$} & \multirow[t]{2}{*}{$\begin{array}{l}80.013 \\
78.499 \\
76 \cdot 266 \\
74.296\end{array}$} & \multirow[t]{2}{*}{$\begin{array}{l}0.44993 \\
0.43852 \\
0.42706 \\
0.41993\end{array}$} & $\begin{array}{l}177.833 \\
179 \cdot 010 \\
178.583 \\
176.925 \\
\end{array}$ & \multirow[b]{2}{*}{0.9183} & $\begin{array}{l}21.1800 \\
17.6731 \\
\end{array}$ \\
\hline & & & $178 \cdot \overline{088}$ & & $\overline{3} \cdot 5069$ \\
\hline
\end{tabular}




\begin{tabular}{|c|c|c|c|c|c|}
\hline Babotanz & $D$ & $J$ & 20 & 8 & $d_{m}$ \\
\hline \multirow[t]{2}{*}{ Buttersaure } & \multirow[t]{2}{*}{$\begin{array}{l}\mathbf{6 7 . 4 4 6} \\
67.030 \\
59.859\end{array}$} & \multirow[t]{2}{*}{$\begin{array}{l}0.74478 \\
0.74433 \\
0.66398\end{array}$} & $\begin{array}{l}90.559 \\
90.054 \\
90.151\end{array}$ & \multirow[b]{2}{*}{0.9633} & \multirow[b]{2}{*}{4.5465} \\
\hline & & & 90.251 & & \\
\hline \multirow{2}{*}{$\begin{array}{c}\text { Buttersaure } \\
\text { in Wasser } \\
a=35 \cdot 090 \%\end{array}$} & \multirow[t]{2}{*}{$\begin{array}{l}69 \cdot 530 \\
69 \cdot 649 \\
67 \cdot 111\end{array}$} & \multirow[t]{2}{*}{$\begin{array}{l}0.69723 \\
0.70648 \\
0.68307\end{array}$} & $\begin{array}{l}99.724 \\
98.586 \\
98.250\end{array}$ & \multirow[b]{2}{*}{1.0042} & $\begin{array}{r}13.6142 \\
9.0636\end{array}$ \\
\hline & & & 98.853 & & 4.5506 \\
\hline \multirow[t]{2}{*}{$\begin{array}{l}\text { II. } \\
a=24.502 \%\end{array}$} & \multirow[t]{2}{*}{$\begin{array}{l}69 \cdot 618 \\
67.851 \\
68 \cdot 347\end{array}$} & \multirow[t]{2}{*}{$\begin{array}{l}0.69425 \\
0.68582 \\
0.68607\end{array}$} & $\begin{array}{r}100.276 \\
98.934 \\
99.621 \\
\end{array}$ & \multirow[b]{2}{*}{1.0056} & $\begin{array}{l}19 \cdot 6192 \\
15 \cdot 0970\end{array}$ \\
\hline & & & $99 \cdot 610$ & & 4.5222 \\
\hline \multirow{2}{*}{$\begin{array}{l}\text { III. } \\
a=12.6299 \%\end{array}$} & \multirow[t]{2}{*}{$\begin{array}{l}65.479 \\
70.362 \\
68.089\end{array}$} & \multirow[t]{2}{*}{$\begin{array}{l}0.65459 \\
0.69851 \\
0.67352\end{array}$} & $\begin{array}{l}100.031 \\
100.733 \\
101.093\end{array}$ & \multirow[b]{2}{*}{1.0069} & $\begin{array}{l}38.3972 \\
33.8951\end{array}$ \\
\hline & & & 100.619 & & 4.5021 \\
\hline \multirow{2}{*}{$\begin{array}{c}\text { Buttersdure } \\
\text { in Benzol } \\
a=34.1865 \%\end{array}$} & \multirow[t]{2}{*}{$\begin{array}{l}123 \cdot 19 \\
119 \cdot 25 \\
119 \cdot 68\end{array}$} & \multirow[t]{2}{*}{$\begin{array}{l}\mathbf{0 . 6 7 6 9 9} \\
0.65150 \\
0.65973\end{array}$} & $\begin{array}{l}181.968 \\
182.996 \\
181.402\end{array}$ & \multirow[b]{2}{*}{0.9059} & $\begin{array}{l}28.538 \\
24.028\end{array}$ \\
\hline & & & $182 \cdot 122$ & & 4.510 \\
\hline \multirow[t]{2}{*}{$a=6.0295 \%$} & \multirow[t]{2}{*}{$\begin{array}{l}148.41 \\
134 \cdot 16 \\
126.95\end{array}$} & \multirow[t]{2}{*}{$\begin{array}{l}0.67713 \\
0.61051 \\
0.58005\end{array}$} & $\begin{array}{l}219.172 \\
219.754 \\
218.863 \\
\end{array}$ & \multirow[b]{2}{*}{0.8866} & $\begin{array}{l}199.062 \\
194.522\end{array}$ \\
\hline & & & $219 \cdot 263$ & & 4.540 \\
\hline \multirow{2}{*}{$\begin{array}{r}\text { Butters\$nre } \\
\text { in Toluol } \\
a=35.9134 \%\end{array}$} & \multirow[t]{2}{*}{$\begin{array}{l}111.25 \\
111.44 \\
111.52\end{array}$} & \multirow[t]{2}{*}{$\begin{array}{l}0.67099 \\
0.66843 \\
0.66654\end{array}$} & $\begin{array}{l}165 \cdot 801 \\
166.716 \\
167 \cdot 323\end{array}$ & \multirow[b]{2}{*}{0.8996} & $\begin{array}{l}25.0281 \\
20.5264\end{array}$ \\
\hline & & & 166.613 & & 4.5017 \\
\hline \multirow[t]{2}{*}{$\begin{array}{l}\text { II. } \\
a=9.7705 \%\end{array}$} & \multirow[t]{2}{*}{$\begin{array}{l}119.93 \\
120.67 \\
110.85\end{array}$} & \multirow[t]{2}{*}{$\begin{array}{l}0.61353 \\
0.61456 \\
0.56592\end{array}$} & $\begin{array}{l}194.949 \\
196.348 \\
195.880 \\
\end{array}$ & \multirow[b]{2}{*}{0.8771} & $\begin{array}{l}110 \cdot 835 \\
106.226\end{array}$ \\
\hline & & & 195.729 & & $4 \cdot 609$ \\
\hline \multirow[t]{2}{*}{$\begin{array}{c}\text { Monochloressig- } \\
\text { săure in Wasser } \\
a=20.4558 \%\end{array}$} & $\begin{array}{l}61.922 \\
60.397 \\
57.186 \\
55.604\end{array}$ & $\begin{array}{l}0.60003 \\
0.58658 \\
0.56152 \\
0.54040\end{array}$ & $\begin{array}{l}103 \cdot 198 \\
102.965 \\
101 \cdot 842 \\
102.894 \\
\end{array}$ & & $\begin{array}{l}24 \cdot 2996 \\
20 \cdot 4605 \\
\end{array}$ \\
\hline & & & 102.725 & 1.0770 & $\mathbf{3 . 8 3 9 1}$ \\
\hline $\begin{array}{c}\text { II. } \\
a=13.2168 \%\end{array}$ & $\begin{array}{l}68.774 \\
63.571 \\
57.713\end{array}$ & $\begin{array}{l}0.67819 \\
0 \cdot 62385 \\
0.56213\end{array}$ & $\begin{array}{l}101.409 \\
101.901 \\
102.669\end{array}$ & & $\begin{array}{l}38.3550 \\
34.5490\end{array}$ \\
\hline & & & 101.993 & 1.0485 & $3 \cdot 8060$ \\
\hline $\begin{array}{c}\text { Monochloressig- } \\
\text { sarare in Benzol } \\
a=8.1816^{\circ}\end{array}$ & $\begin{array}{l}139.54 \\
137.67 \\
132.22\end{array}$ & $\begin{array}{l}0.63439 \\
0.62368 \\
0.60101\end{array}$ & $\begin{array}{l}219.956 \\
220.743 \\
219.994 \\
\end{array}$ & & $\begin{array}{l}154 \cdot 161 \\
150 \cdot 438 \\
\end{array}$ \\
\hline & & & 220.231 & 0.9101 & $3 \cdot 723$ \\
\hline $\begin{array}{c}\text { Monochloressig- } \\
\text { săure in Toluol } \\
a=5.6385 \%\end{array}$ & $\begin{array}{l}120.76 \\
115.91 \\
112.34\end{array}$ & $\begin{array}{l}0.59785 \\
0.57406 \\
0.55389\end{array}$ & $\begin{array}{l}201.987 \\
201.906 \\
202.822\end{array}$ & & $\begin{array}{l}210.562 \\
206.722\end{array}$ \\
\hline$-\quad .0000 \%$ & & & 202.238 & 0.8877 & $\mathbf{3 . 8 4 0}$ \\
\hline
\end{tabular}


Ober die olektromagnet. Drehung der Polarisationeebene einiger Sarren otc. 407

\begin{tabular}{|c|c|c|c|c|c|}
\hline Bubatan: & $D$ & $\boldsymbol{J}$ & 20 & 8 & $d m$ \\
\hline \multirow[t]{2}{*}{$\begin{array}{l}\text { Dichloressig- } \\
\text { sdure }\end{array}$} & \multirow[t]{2}{*}{$\begin{array}{l}75 \cdot 632 \\
74.466 \\
74.560 \\
74.061\end{array}$} & \multirow[t]{2}{*}{$\begin{array}{l}0.67028 \\
0.66389 \\
0.65580 \\
0.66041\end{array}$} & $\begin{array}{l}112 \cdot 837 \\
112 \cdot 165 \\
113 \cdot 694 \\
112 \cdot 145 \\
\end{array}$ & \multirow[b]{2}{*}{1.5488} & \multirow[b]{2}{*}{5.1770} \\
\hline & & & 112.710 & & \\
\hline \multirow{2}{*}{$\begin{array}{c}\text { Dichloressig- } \\
\text { säure in Wasser } \\
a=7.4751 \%\end{array}$} & \multirow[t]{2}{*}{$\begin{array}{l}71.643 \\
71.089 \\
68.468\end{array}$} & \multirow[t]{2}{*}{$\begin{array}{l}0.69774 \\
0.69768 \\
0.67382\end{array}$} & $\begin{array}{l}102 \cdot 678 \\
101 \cdot 894 \\
101 \cdot 612 \\
\end{array}$ & \multirow[b]{2}{*}{1.0322} & $\begin{array}{l}94.0984 \\
\mathbf{8 8 . 9 0 3 5} \\
\end{array}$ \\
\hline & & & 102.061 & & 5.1949 \\
\hline \multirow[t]{2}{*}{$\begin{array}{c}\text { Dichloressig- } \\
\text { săure in Benzol } \\
a=24.4065 \%\end{array}$} & \multirow[t]{2}{*}{$\begin{array}{l}138.17 \\
130.14 \\
128.62\end{array}$} & \multirow[t]{2}{*}{$\begin{array}{l}0.65441 \\
0.61254 \\
0.60833\end{array}$} & $\begin{array}{l}211.134 \\
212.461 \\
211.431 \\
\end{array}$ & \multirow[b]{2}{*}{0.9860} & $\begin{array}{r}62.5755 \\
57.3375 \\
\end{array}$ \\
\hline & & & 211.675 & & 5.2380 \\
\hline \multirow[t]{2}{*}{$\begin{array}{c}\text { Dichloressig- } \\
\text { săure in Toluol } \\
a=24.6976 \%\end{array}$} & \multirow[t]{2}{*}{$\begin{array}{l}136 \cdot 65 \\
135 \cdot 45 \\
126 \cdot 69 \\
123 \cdot 14\end{array}$} & \multirow[t]{2}{*}{$\begin{array}{l}0.71551 \\
0.70663 \\
0.65868 \\
0.64188\end{array}$} & $\begin{array}{l}190.984 \\
181.676 \\
192.349 \\
191.850 \\
\end{array}$ & \multirow[b]{2}{*}{0.9761} & $\begin{array}{l}56.5748 \\
51.4121\end{array}$ \\
\hline & & & 191.715 & & $5 \cdot 1627$ \\
\hline \multirow[t]{2}{*}{$\begin{array}{c}\text { II. } \\
a=7.5869 \%\end{array}$} & \multirow[t]{2}{*}{$\begin{array}{l}81 \cdot 830 \\
77.663 \\
74.099\end{array}$} & \multirow[t]{2}{*}{$\begin{array}{l}0.40780 \\
0.38441 \\
0.36643\end{array}$} & $\begin{array}{l}200 \cdot 662 \\
202 \cdot 033 \\
202.213\end{array}$ & \multirow[b]{2}{*}{0.8980} & $\begin{array}{l}210.5465 \\
205.3910\end{array}$ \\
\hline & & & 201.636 & & 5.1555 \\
\hline
\end{tabular}

Wenn nun auch bezüglich der Fettsäuren der Einwand erhoben werden könnte, dass dieselben in wässrigen Lösungen einer zu wenig weit gehenden Dissociation verfallen, so trifft dieser Einwand für dio verhältnismässig stark dissociierten Chloressigsäuren nicht mohr zu. Es wurde nun, um stark dissociierte Verbindungen in derselben Richtung zu untersuchen, die elektromagnetische Drehung für einige Salze in wässrigen und methylalkoholischen Lösungen ermittelt. Es wurden solche Salze gewählt, die sich ziemlich leicht in Methylalkohol lösen: Jodkalium, Bromnatrium, Ammoniumnitrat und Baryumbromid. Die Konzentration der Lösungen für das erste Salz wurde durch Mischen gewogener Mengen bestimmt. Gleichfalls sollte auch in den anderen Fällen verfahren werden, allein die Lösungen erwiesen sich als trübe, so dass eine genaue Einstellung des Analysators bei der $800 \mathrm{~mm}$ betragenden Flüssigkeitssäule nicht erlangt werden konnte. Nach dem Filtrieren waren die Mischungen brauchbar; da aber nach den Untersuchungen von Landolt die Konzentration bei einer solchen Operation sich ändert, so musste der Salzgehalt der Lösungen von neuem bestimmt werden. Dies geschah bei den Solutionen des Bromnatriums durch Eindampfen gewogener Flüssigkeitsmengen and Bestimmung des Rückstandes im Platintiegel. Für die Lösungen des Ammoniumnitrates wrurde 
die Konzentration durch Bestimmung des Ammoniaks, für die des Baryumnitrats durch Brombestimmungen nach den üblichen Methoden ermittelt. Die Resultate dieser Analysen waren folgende.

$\mathrm{NaBr}$ in $\mathrm{H}_{2} \mathrm{O}$.

$10.2346 \mathrm{~g}$ Lösung gaben $3.0402 \mathrm{~g} \mathrm{NaBr} \quad a=29.705 \%$, $\mathrm{NaBr}$ in $\mathrm{CH}_{3} . \mathrm{OH}$ Lösung (1).
$8.7764 \mathrm{~g}$ Lösung $=0.6462 \mathrm{~g} \mathrm{NaBr}$
$15.6544, "=1.1524, "$,
$7.3630 \%$
$7.3615 \%$
$a=7.3622 \%$

$\mathrm{NaBr}$ in $\mathrm{CH}_{3} . \mathrm{OH}$ Lösung (2).

$10.7010 \mathrm{~g}$ Lösung $=1.0224 \mathrm{~g} \mathrm{NaBr} \quad 9.5542 \% \quad a=9.5524 \%$ $12.8433, \quad "=1.2266, \quad " \quad 9.5505 \%$

$\mathrm{NH}_{\mathbf{4}} \cdot \mathrm{NO}_{\mathrm{s}}$ in $\mathrm{H}_{\mathrm{q}} \mathrm{O}$. Lösung (1).

$5.5405 \mathrm{~g}$ Lösung $=0.27649 \mathrm{~g} \mathrm{NH}_{3} \quad a=23.484 \%$

$\mathrm{NH}_{4} \mathrm{NO}_{3}$ in $\mathrm{H}_{2} \mathrm{O}$. Lösung (2).

$6.2833 \mathrm{~g}$ Lösung $=0.18772 \mathrm{~g} \mathrm{NH_{3 }} \quad a=14.059 \%$

$\mathrm{NH}_{4} \cdot \mathrm{NO}_{3}$ in $\mathrm{CH}_{3} . \mathrm{OH}$. Lösung (1).

$4.4378 \mathrm{~g}$ Lösung $=0.05359 \mathrm{~g} \mathrm{NH_{3 }} \quad a=5.683 \%$

$\mathrm{NH}_{4} \mathrm{NO}_{3}$ in $\mathrm{CH}_{3} . \mathrm{OH}$. Lösung (2)

$3.9631 \mathrm{~g}$ Lösung $=0.11160 \mathrm{~g} \mathrm{NH} H_{3} \quad a=13.252 \%$

$\mathrm{BaBr}_{8}$ in $\mathrm{H}_{8} \mathrm{O}$.

$18.6850 \mathrm{~g}$ Lösung $=3.1926 \mathrm{~g} \mathrm{AgBr} \quad a=13.497 \%$

$\mathrm{BaBr}_{\mathrm{z}}$ in $\mathrm{CH}_{3} . \mathrm{OH}$. Lösung (1)

$14.4700 \mathrm{~g}$ Lösung $=3.2645 \mathrm{~g} \mathrm{AgBr}=17.820 \% a=17.847 \%$

$9.4869, \quad "=2.1469 \mathrm{~g} \mathrm{AgBr}=17.875 \%$

$\mathrm{BaBr}_{2}$ in $\mathrm{CH}_{3} . \mathrm{OH}$. Lösung (2).

$11.6089 \mathrm{~g}$ Lösung $=1.8451 \mathrm{~g} \mathrm{AgBr}$,

$$
a=12.554 \% \text {. }
$$

Die Zahlen über die elektromagnetische Drehung dieser Salze sind in der Tabelle VI niedergelegt. Gleichzeitig hatte Herr cand. phys. Ed. Schroeder die Güte, für diese Lösungen das Leitungsvermögen zu bestimmen. Ich möchte nicht versäumen, an dieser Stelle ihm hierfür meinen besten Dank auszusprechen. In der Tabelle VII, die die von Herrn Schroeder gefundenen Daten enthält, ist in der ersten Rubrik der mit Hilfe der Wheatstoneschen Brücke ermittelte Widerstand angegeben. Durch Division der Kapazität des Gefässes, die zu 21886.4 gefunden wurde, durch den Widerstand $w$ erhielt man das spezifische Leitrermögen $k$. Daraus wurde die molekulare Leitfähigkeit $l_{m}$ berechnet, indem man $k$ mit der Anzahl Liter $v$ multipliziert, in denen eine Grammmolekel Substanz gelöst ist. 
Ober die elektromagnet. Drehnng der Polarisationsebene einiger Sauren etc. 409 Tabelle VI.

\begin{tabular}{|c|c|c|c|c|c|}
\hline Subatanz & $D$ & $\boldsymbol{J}$ & 20 & 8 & $d_{m}$ \\
\hline \multirow{2}{*}{$\begin{array}{c}\text { Jodkalium } \\
\text { in Wasser } \\
a=39.0736 \%\end{array}$} & \multirow[t]{2}{*}{$\begin{array}{l}147.02 \\
146.73 \\
143.76\end{array}$} & \multirow[t]{2}{*}{$\begin{array}{l}0.74578 \\
0.74239 \\
0.72631\end{array}$} & $\begin{array}{l}197.042 \\
197.639 \\
197.927\end{array}$ & \multirow[b]{2}{*}{1.3835} & $\begin{array}{l}33 \cdot 3553 \\
14.4120\end{array}$ \\
\hline & & & 197.536 & & 18.9433 \\
\hline \multirow[t]{2}{*}{$\begin{array}{c}\text { II. } \\
a=15.4670 \%\end{array}$} & \multirow[t]{2}{*}{$\begin{array}{l}94 \cdot 200 \\
88 \cdot 105 \\
90 \cdot 345\end{array}$} & \multirow[t]{2}{*}{$\begin{array}{l}0.71140 \\
0.66293 \\
0.68327\end{array}$} & $\begin{array}{l}132.416 \\
132 \cdot 902 \\
132 \cdot 224\end{array}$ & \multirow[b]{2}{*}{1.1258} & $\begin{array}{r}69.4665 \\
50.5152 \\
\end{array}$ \\
\hline & & & $132 \cdot 514$ & & 18.9513 \\
\hline \multirow[t]{2}{*}{$\begin{array}{c}\text { Jodkalium in } \\
\text { Methylalkohol } \\
a=9.1409 \%\end{array}$} & \multirow[t]{2}{*}{$\begin{array}{l}63.817 \\
63.231 \\
61.639 \\
62.487\end{array}$} & \multirow[t]{2}{*}{$\begin{array}{l}0.73000 \\
0.72686 \\
0.70442 \\
0.71606\end{array}$} & $\begin{array}{l}87 \cdot 448 \\
86 \cdot 992 \\
87.504 \\
87.265\end{array}$ & \multirow[b]{2}{*}{0.8633} & $\begin{array}{r}100.984 \\
81.977\end{array}$ \\
\hline & & & 87.302 & & 19.007 \\
\hline \multirow[t]{2}{*}{$\begin{array}{c}\text { II. } \\
a=8.2279 \%\end{array}$} & \multirow[t]{2}{*}{$\begin{array}{l}60.066 \\
59.348 \\
60.219\end{array}$} & \multirow[t]{2}{*}{$\begin{array}{l}0.70639 \\
0.69078 \\
0.70215\end{array}$} & $\begin{array}{l}85.033 \\
85.914 \\
85.764\end{array}$ & \multirow[b]{2}{*}{0.8560} & $\begin{array}{r}110.899 \\
91.989\end{array}$ \\
\hline & & & 85.570 & & 18.910 \\
\hline \multirow[t]{2}{*}{$\begin{array}{r}\text { Bromnatrinm } \\
\text { in Wasser } \\
a=29.705 \%\end{array}$} & \multirow[t]{2}{*}{$\begin{array}{l}103 \cdot 628 \\
103 \cdot 706 \\
103 \cdot 148 \\
103 \cdot 752\end{array}$} & \multirow[t]{2}{*}{$\begin{array}{l}0.67979 \\
0.67772 \\
0.67876 \\
0.67673\end{array}$} & $\begin{array}{l}152 \cdot 443 \\
153 \cdot 021 \\
151.967 \\
153.314 \\
\end{array}$ & \multirow[b]{2}{*}{1.2793} & $\begin{array}{l}22.7566 \\
13.5714\end{array}$ \\
\hline & & & 152.686 & & $9 \cdot 1852$ \\
\hline \multirow[t]{2}{*}{$\begin{array}{c}\text { Bromnatrium } \\
\text { in Methylalkobol } \\
a=7.3622 \%\end{array}$} & \multirow[t]{2}{*}{$\begin{array}{l}\mathbf{4 8 . 9 1 9} \\
\mathbf{5 1 . 1 3 5} \\
\mathbf{5 1 . 3 8 8} \\
\mathbf{5 0 . 8 9 9}\end{array}$} & \multirow[t]{2}{*}{$\begin{array}{l}0.59929 \\
0.62334 \\
0.63071 \\
0.62544\end{array}$} & $\begin{array}{l}81.629 \\
82.034 \\
81.477 \\
81.381\end{array}$ & \multirow[b]{2}{*}{0.8535} & $\begin{array}{l}73 \cdot 5827 \\
64.3906\end{array}$ \\
\hline & & & 81.630 & & $9 \cdot 1921$ \\
\hline \multirow[t]{2}{*}{$\begin{array}{c}\text { II. } \\
a=9.5524 \%\end{array}$} & \multirow[t]{2}{*}{$\begin{array}{l}62.050 \\
61 \cdot 255 \\
50.124\end{array}$} & \multirow[t]{2}{*}{$\begin{array}{l}0.73270 \\
0.72464 \\
0.58650\end{array}$} & $\begin{array}{l}84.687 \\
84.531 \\
85.463\end{array}$ & \multirow[b]{2}{*}{0.8730} & $\begin{array}{l}57.6582 \\
48.4536\end{array}$ \\
\hline & & & 84.894 & & $9 \cdot 2046$ \\
\hline \multirow[t]{2}{*}{$\begin{array}{c}\text { Ammoniumnitrat } \\
\text { in Wasser } \\
a=23.48 \%\end{array}$} & \multirow[t]{2}{*}{$\begin{array}{l}61.512 \\
61.445 \\
63.054\end{array}$} & \multirow[t]{2}{*}{$\begin{array}{l}0.62652 \\
0.61190 \\
0.64423\end{array}$} & $\begin{array}{l}98.181 \\
98 \cdot 131 \\
97.876 \\
\end{array}$ & & $\begin{array}{l}16 \cdot 663 \\
14.513\end{array}$ \\
\hline & & & 98.063 & $1 \cdot 1024$ & $2 \cdot 150$ \\
\hline \multirow[t]{2}{*}{$\begin{array}{l}\text { II. } \\
a=14.06 \%\end{array}$} & \multirow[t]{2}{*}{$\begin{array}{l}\mathbf{5 8 . 4 7 0} \\
\mathbf{5 9 . 5 7 8} \\
\mathbf{5 7 . 8 9 4}\end{array}$} & \multirow[t]{2}{*}{$\begin{array}{l}0.58636 \\
0.59686 \\
0.57862\end{array}$} & $\begin{array}{r}99.716 \\
99.819 \\
100.055 \\
\end{array}$ & & $\begin{array}{r}29.3982 \\
27.2280 \\
\end{array}$ \\
\hline & & & 99.863 & 1.0629 & 2.1702 \\
\hline \multirow[t]{2}{*}{$\begin{array}{c}\text { Ammoniumnitrat } \\
\text { in Methylalkohol } \\
a=5.68 \%\end{array}$} & \multirow[t]{2}{*}{$\begin{array}{l}44 \cdot 193 \\
42.990 \\
43 \cdot 016 \\
42.973\end{array}$} & $\begin{array}{l}0.59584 \\
0.58778 \\
0.59706 \\
0.58916\end{array}$ & $\begin{array}{l}74 \cdot 169 \\
73 \cdot 138 \\
72 \cdot 046 \\
72.938 \\
\end{array}$ & & $\begin{array}{r}68.1720 \\
65.9640 \\
\end{array}$ \\
\hline & & & 73.073 & 0.8297 & 2.2080 \\
\hline
\end{tabular}




\begin{tabular}{|c|c|c|c|c|c|}
\hline 8ubetanx & $D$ & $J$ & 20 & 8 & $d_{m}$ \\
\hline \multirow[t]{2}{*}{$a=13.25 \%$} & \multirow[t]{2}{*}{$\begin{array}{l}42.986 \\
43.745 \\
42.854\end{array}$} & \multirow[t]{2}{*}{$\begin{array}{l}0.59001 \\
0.59928 \\
0.58753\end{array}$} & $\begin{array}{l}72 \cdot 856 \\
72.996 \\
72.939\end{array}$ & \multirow[b]{2}{*}{0.8584} & $\begin{array}{l}28.202 \\
26.018 \\
\end{array}$ \\
\hline & & & 72.930 & & 2.184 \\
\hline \multirow[t]{2}{*}{$\begin{array}{c}\text { Brombaryum } \\
\text { in Wasser } \\
a=13.497 \%\end{array}$} & \multirow[t]{2}{*}{$\begin{array}{l}65.697 \\
65.213 \\
65.307 \\
65.144\end{array}$} & \multirow[t]{2}{*}{$\begin{array}{l}0.56129 \\
0.55958 \\
0.56300 \\
0.56127\end{array}$} & $\begin{array}{l}117.048 \\
116.539 \\
115.998 \\
116.064\end{array}$ & \multirow[b]{2}{*}{1.0315} & $\begin{array}{l}124.491 \\
105.990\end{array}$ \\
\hline & & & 116.412 & & 18.501 \\
\hline \multirow[t]{2}{*}{$\begin{array}{c}\text { Brombaryum } \\
\text { in Methylalkohol } \\
a=17.847 \%\end{array}$} & \multirow[t]{2}{*}{$\begin{array}{l}62.061 \\
59.768 \\
57.756 \\
\mathbf{5 9 . 5 8 7}\end{array}$} & \multirow[t]{2}{*}{$\begin{array}{l}0.67716 \\
0.64122 \\
0.62686 \\
0.64758\end{array}$} & $\begin{array}{l}91 \cdot 649 \\
91.087 \\
92 \cdot 135 \\
92.014\end{array}$ & \multirow[b]{2}{*}{0.9701} & $\begin{array}{l}86.5173 \\
67.9234 \\
\end{array}$ \\
\hline & & & 91.721 & & 18.5939 \\
\hline \multirow[t]{2}{*}{$\begin{array}{c}\text { II. } \\
a=12.554 \%\end{array}$} & \multirow[t]{2}{*}{$\begin{array}{l}50.402 \\
50.577 \\
49.855 \\
49.780\end{array}$} & \multirow[t]{2}{*}{$\begin{array}{l}0.59055 \\
0.58920 \\
0.59183 \\
0.58675\end{array}$} & $\begin{array}{l}85 \cdot 347 \\
85.841 \\
84 \cdot 239 \\
84.840 \\
\end{array}$ & \multirow[b]{2}{*}{0.9125} & $\begin{array}{l}121.272 \\
102.778 \\
\end{array}$ \\
\hline & & & 85.067 & & 18.494 \\
\hline
\end{tabular}

Tabelle VII.

\begin{tabular}{|c|c|c|c|c|}
\hline Substanz & $w$ & $k$ & $v$ & $l_{m}$ \\
\hline \multirow{3}{*}{$\begin{array}{l}\text { Jodkalium in Wasser, nac } \\
\text { Jodkalium in Methylalkohol }\end{array}$} & \multirow{3}{*}{$\begin{array}{l}\text { Kohlrausch } \\
1032.47 \\
1034.68 \\
1033.28\end{array}$} & Messungen & \multirow[t]{3}{*}{$\ldots \ldots$} & \multirow[t]{2}{*}{103} \\
\hline & & $\begin{array}{l}21 \cdot 194 \\
21 \cdot 149 \\
21 \cdot 178\end{array}$ & & \\
\hline & & 21.174 & & 44.541 \\
\hline \multirow[t]{2}{*}{ II. } & \multirow[t]{2}{*}{$\begin{array}{l}1134 \cdot 16 \\
1130 \cdot 14 \\
1127 \cdot 29\end{array}$} & $\begin{array}{l}19 \cdot 294 \\
19 \cdot 362 \\
19 \cdot 411\end{array}$ & \multirow[b]{2}{*}{$2 \cdot 3568$} & \multirow[b]{2}{*}{$45 \cdot 620$} \\
\hline & & $19 \cdot \overline{356}$ & & \\
\hline \multirow[t]{2}{*}{ Bromnatrium in Wasser } & \multirow[t]{2}{*}{$\begin{array}{l}115 \cdot 16 \\
114 \cdot 76 \\
114 \cdot 71\end{array}$} & $\begin{array}{l}190.05 \\
190.71 \\
190.80\end{array}$ & \multirow[b]{2}{*}{$0 \cdot 2710$} & \multirow[b]{2}{*}{51.639} \\
\hline & & $190 \cdot \overline{52}$ & & \\
\hline \multirow[t]{2}{*}{$\begin{array}{l}\text { Bromnatrium } \\
\quad \text { in Methylalkohol }\end{array}$} & \multirow[t]{2}{*}{$\begin{array}{l}1165 \cdot 52 \\
1167 \cdot 48 \\
1174 \cdot 25\end{array}$} & $\begin{array}{l}18 \cdot 774 \\
18 \cdot 743 \\
18 \cdot 635\end{array}$ & \multirow[b]{2}{*}{1.6393} & \multirow[b]{2}{*}{30.682} \\
\hline & & 18.717 & & \\
\hline \multirow[t]{2}{*}{ II. } & \multirow[t]{2}{*}{$\begin{array}{l}985 \cdot 87 \\
988 \cdot 16 \\
992 \cdot 63\end{array}$} & $\begin{array}{l}22 \cdot 196 \\
22 \cdot 145 \\
22 \cdot 045\end{array}$ & \multirow[b]{2}{*}{1.2351} & \multirow[b]{2}{*}{27.333} \\
\hline & & 22.129 & & \\
\hline \multirow[t]{2}{*}{$\begin{array}{l}\text { Ammoniumnitrat } \\
\text { in Wasser }\end{array}$} & \multirow[t]{2}{*}{$\begin{array}{l}98.404 \\
98.519 \\
98.834\end{array}$} & $\begin{array}{l}222.41 \\
222 \cdot 15 \\
221 \cdot 45\end{array}$ & \multirow[b]{2}{*}{0.3090} & \multirow[b]{2}{*}{$68 \cdot 600$} \\
\hline & & 222.00 & & \\
\hline
\end{tabular}


Ubber die elektromagnet. Drehung der Polariantionsebene einiger Săuren etc. 411

\begin{tabular}{|c|c|c|c|c|}
\hline $\operatorname{sab} \sin z$ & 20 & $\boldsymbol{k}$ & 0 & $I_{m}$ \\
\hline \multirow[t]{2}{*}{ II. $\cdot$} & \multirow[t]{2}{*}{$\begin{array}{l}145.54 \\
145.46 \\
145.52\end{array}$} & $\begin{array}{l}150-38 \\
150 \cdot 46 \\
150-40\end{array}$ & \multirow[b]{2}{*}{0.5356} & \multirow[b]{2}{*}{80.523} \\
\hline & & 150.41 & & \\
\hline \multirow[t]{2}{*}{$\begin{array}{l}\text { Ammoniumnitrat } \\
\text { in Methylalkohol }\end{array}$} & \multirow[t]{2}{*}{$\begin{array}{l}1028 \cdot 33 \\
1028.71 \\
1027 \cdot 15\end{array}$} & $\begin{array}{l}21.284 \\
21.276 \\
21.308\end{array}$ & \multirow[b]{2}{*}{1.6975} & \multirow[b]{2}{*}{$36 \cdot 137$} \\
\hline & & 21.289 & & \\
\hline \multirow[t]{2}{*}{ II. } & \multirow[t]{2}{*}{$\begin{array}{l}458 \cdot 97 \\
458.74 \\
458.84\end{array}$} & $\begin{array}{l}47 \cdot 686 \\
47 \cdot 710 \\
47 \cdot 699\end{array}$ & \multirow[b]{2}{*}{0.7032} & \multirow[b]{2}{*}{33.543} \\
\hline & & 47.698 & & \\
\hline \multirow[t]{2}{*}{ Brombaryum in Wasser } & \multirow[t]{2}{*}{$\begin{array}{l}306.48 \\
306 \cdot 32 \\
306 \cdot 55\end{array}$} & $\begin{array}{l}71.412 \\
71.448 \\
71.390\end{array}$ & \multirow[b]{2}{*}{1.9448} & \multirow[b]{2}{*}{138.89} \\
\hline & & 71.416 & & \\
\hline \multirow[t]{2}{*}{$\begin{array}{l}\text { Brombaryum } \\
\text { in Methylalkohol }\end{array}$} & \multirow[t]{2}{*}{$\begin{array}{l}1068 \cdot 19 \\
1067.21 \\
1068 \cdot 19\end{array}$} & $\begin{array}{l}20.489 \\
20.508 \\
20.489\end{array}$ & \multirow[b]{2}{*}{1.7154} & \multirow[b]{2}{*}{$35 \cdot 156$} \\
\hline & & 20.495 & & \\
\hline \multirow[t]{2}{*}{ II. } & \multirow[t]{2}{*}{$\begin{array}{l}\mathbf{1 3 5 8 . 7} \\
1359.7 \\
1359 \cdot 3\end{array}$} & $\begin{array}{l}16 \cdot 108 \\
16 \cdot 134 \\
16 \cdot 138\end{array}$ & \multirow[b]{2}{*}{2.5925} & \multirow[b]{2}{*}{41.810} \\
\hline & & 16.127 & & \\
\hline
\end{tabular}

Die in der Tabelle VI enthaltenen Zahlen für die Molekulard́rehungen in wässrigen und alkoholischen Lösungen stimmen sehr gut miteinander überein, so dass man wohl ihre Identität annehmen kann. $\mathrm{Zu}$ einem ganz anderen Resultat gelangt man beim Vergleich der für das Molekularleitrermögen ermittelten Zahlen. Dieselben weichen für die beiden Lösungsmittel stark voneinander ab; die Salze haben im Methylalkohol durchweg ein kleineres Molekularleitvermögen als im Wasser, was auf eine geringere Dissociation hinweist. So ergiebt sich denn auch aus der Untersuchung dieser Salze, dass der Einfluss der Dissociation auf die elektromagnetische Drehung, falls er besteht, ein zu geringer ist, um mit Hilfe der üblichen Messmethoden nachgewiesen werden zu können.

Es wurden nun schliesslich noch die elektromagnetischen Drehungen einiger substituierten Fettsäuren sowio die Chlor- und Bromderivate einiger Kohlenwasserstoffe ermittelt, für welch letztere Schönrock die Rotation bereits gemessen hat. Die Resultate der diesbezüglichen Versuche sind in der folgenden Tabelle zusammengestellt. Für die Monound Dichloressigsäure sind die Zahlen auf den Seiten 406 und 407 angegeben worden. 
Tabelle VIII.

\begin{tabular}{|c|c|c|c|c|c|}
\hline Substanz & $D$ & $J$ & 20 & 8 & $d_{m i}$ \\
\hline \multirow[t]{2}{*}{$\begin{array}{r}\text { Trichloressig- } \\
\text { saure in Wasser } \\
a=20.6568 \%\end{array}$} & \multirow[t]{2}{*}{$\begin{array}{l}55.328 \\
49.021 \\
\mathbf{4 8 \cdot 1 5 2}\end{array}$} & \multirow[t]{2}{*}{$\begin{array}{l}0.52599 \\
0.46113 \\
0.45878\end{array}$} & $\begin{array}{l}105 \cdot 189 \\
106 \cdot 308 \\
104 \cdot 955\end{array}$ & \multirow[b]{2}{*}{1.1094} & $\begin{array}{l}41.5011 \\
34.9669\end{array}$ \\
\hline & & & 105.484 & & 6.5342 \\
\hline \multirow[t]{2}{*}{$\begin{array}{c}\text { II. } \\
a=10.4288 \%\end{array}$} & \multirow[t]{2}{*}{$\begin{array}{l}67 \cdot 326 \\
65 \cdot 193 \\
61.236\end{array}$} & \multirow[t]{2}{*}{$\begin{array}{l}0.65262 \\
0.63693 \\
0.58913\end{array}$} & $\begin{array}{l}103 \cdot 163 \\
102 \cdot 357 \\
103 \cdot 942\end{array}$ & \multirow[b]{2}{*}{1.0528} & $\begin{array}{l}84.7143 \\
78.1885\end{array}$ \\
\hline & & & $103 \cdot 154$ & & 6.5258 \\
\hline \multirow{2}{*}{$\begin{array}{c}\text { Trichlorbutter- } \\
\text { săure in Wasser } \\
a=20.2963 \%\end{array}$} & \multirow[t]{2}{*}{$\begin{array}{l}64 \cdot 179 \\
62 \cdot 833 \\
62 \cdot 208\end{array}$} & \multirow[t]{2}{*}{$\begin{array}{l}0.61469 \\
0.60001 \\
0.59704\end{array}$} & $\begin{array}{l}104 \cdot 409 \\
104 \cdot 720 \\
104 \cdot 193\end{array}$ & \multirow[b]{2}{*}{1.0796} & $\begin{array}{l}50.203 \\
\mathbf{4 1 . 7 6 0}\end{array}$ \\
\hline & & & 104.441 & & 8.443 \\
\hline \multirow[t]{2}{*}{$\begin{array}{c}\text { II. } \\
a=32.6527 \%\end{array}$} & \multirow[t]{2}{*}{$\begin{array}{l}57.861 \\
57.424 \\
57.591\end{array}$} & \multirow[t]{2}{*}{$\begin{array}{l}0.54356 \\
0.53022 \\
0.53478\end{array}$} & $\begin{array}{l}106.939 \\
108.302 \\
107.691\end{array}$ & \multirow[b]{2}{*}{$1 \cdot 1335$} & $\begin{array}{l}30.6341 \\
21.9346\end{array}$ \\
\hline & & & 107.644 & & 8.6995 \\
\hline \multirow[t]{2}{*}{$\begin{array}{c}\text { Monobromessig- } \\
\text { săure in Wasser } \\
a=30.9545 \%\end{array}$} & \multirow[t]{2}{*}{$\begin{array}{l}64 \cdot 681 \\
66 \cdot 312 \\
66 \cdot 376\end{array}$} & \multirow[t]{2}{*}{$\begin{array}{l}0.58773 \\
0.59715 \\
0.59596\end{array}$} & $\begin{array}{l}110.052 \\
111.046 \\
111.377\end{array}$ & & $\begin{array}{l}22.9710 \\
17.2631\end{array}$ \\
\hline & & & 110.825 & 1.1913 & 5.7079 \\
\hline \multirow[t]{2}{*}{$a=19.9427 \%$} & \multirow[t]{2}{*}{$\begin{array}{l}49 \cdot 696 \\
50 \cdot 976 \\
48 \cdot 327 \\
48 \cdot 247\end{array}$} & \multirow[t]{2}{*}{$\begin{array}{l}0.46465 \\
0.47763 \\
0.45021 \\
0.44668\end{array}$} & $\begin{array}{l}106 \cdot 954 \\
106 \cdot 706 \\
107 \cdot 344 \\
108 \cdot 012\end{array}$ & \multirow[b]{2}{*}{1.1161} & $\begin{array}{l}36.8309 \\
31.0690\end{array}$ \\
\hline & & & $107 \cdot 254$ & & $5 \cdot 7619$ \\
\hline \multirow{2}{*}{$\begin{array}{r}\text { Tribromessig- } \\
\text { 8ăure in Wasser } \\
a=32.0282 \%\end{array}$} & \multirow[t]{2}{*}{$\begin{array}{l}59.130 \\
63.196 \\
63.304\end{array}$} & \multirow[t]{2}{*}{$\begin{array}{l}0.49721 \\
0.53787 \\
0.53644\end{array}$} & $\begin{array}{l}118.923 \\
117.495 \\
118.007\end{array}$ & \multirow[b]{2}{*}{1.2735} & $\begin{array}{l}47.2811 \\
34.9769\end{array}$ \\
\hline & & & $118 \cdot 142$ & & $12 \cdot 3042$ \\
\hline \multirow[t]{2}{*}{$\begin{array}{c}\text { II. } \\
a=20.5283 \%\end{array}$} & \multirow[t]{2}{*}{$\begin{array}{l}71.982 \\
77.755 \\
68.788\end{array}$} & \multirow[t]{2}{*}{$\begin{array}{l}0.65183 \\
0.68935 \\
0.62610\end{array}$} & $\begin{array}{l}110.430 \\
112.789 \\
109.868\end{array}$ & \multirow[b]{2}{*}{1.1602} & $\begin{array}{l}76.0966 \\
63.8036\end{array}$ \\
\hline & & & 111.029 & & $\overline{12} \cdot \overline{2930}$ \\
\hline \multirow[t]{2}{*}{$\begin{array}{c}\text { III. } \\
a=8.5535 \%\end{array}$} & \multirow[t]{2}{*}{$\begin{array}{l}61 \cdot 564 \\
39 \cdot 269 \\
62 \cdot 139\end{array}$} & \multirow[t]{2}{*}{$\begin{array}{l}0.58970 \\
0.37673 \\
0.58949\end{array}$} & $\begin{array}{l}104 \cdot 639 \\
104 \cdot 235 \\
105 \cdot 413\end{array}$ & & $\begin{array}{l}188.500 \\
176.201\end{array}$ \\
\hline & & & 104.762 & 1.0606 & $12 \cdot 299$ \\
\hline \multirow[t]{2}{*}{ Äthylenbromid } & $\begin{array}{l}120 \cdot 11 \\
115 \cdot 15 \\
116 \cdot 65\end{array}$ & $\begin{array}{l}0.58586 \\
0.55913 \\
0.57121\end{array}$ & $\begin{array}{l}205.016 \\
205 \cdot 952 \\
204 \cdot 218\end{array}$ & & \\
\hline & & & 205.062 & $2 \cdot 1843$ & 9.7021 \\
\hline Amylenbromid & $\begin{array}{l}98.124 \\
99.771 \\
96.498\end{array}$ & $\begin{array}{l}0.58696 \\
0.59673 \\
0.57981\end{array}$ & $\begin{array}{l}167 \cdot 172 \\
167 \cdot 196 \\
166.432\end{array}$ & & \\
\hline & & & 166.933 & 1.6670 & 12.6684 \\
\hline
\end{tabular}


Ober die elektromagnet. Drehung der Polarisationsebene einiger Sauren etc. 413

\begin{tabular}{|c|c|c|c|c|c|}
\hline Bubetane & $D$ & $\boldsymbol{J}$ & 20 & 8 & $d_{m}$ \\
\hline \multirow[t]{2}{*}{ Amylchlorid } & \multirow[t]{2}{*}{$\begin{array}{l}52 \cdot 559 \\
56 \cdot 284 \\
54 \cdot 232 \\
55 \cdot 134\end{array}$} & \multirow[t]{2}{*}{$\begin{array}{l}0.48638 \\
0.52214 \\
0.51071 \\
0.51247\end{array}$} & $\begin{array}{l}108.059 \\
107.796 \\
106 \cdot 189 \\
107.585\end{array}$ & \multirow[b]{2}{*}{0.8739} & \multirow[b]{2}{*}{$7 \cdot 1848$} \\
\hline & & & 107.407 & & \\
\hline \multirow[t]{2}{*}{$\begin{array}{l}\text { Amylchlorid } \\
\text { (tert.) }\end{array}$} & \multirow[t]{2}{*}{$\begin{array}{l}63.567 \\
66 \cdot 609 \\
65.227 \\
64.827\end{array}$} & \multirow[t]{2}{*}{$\begin{array}{l}0.60386 \\
0.62705 \\
0.62420 \\
0.61382\end{array}$} & $\begin{array}{l}105 \cdot 269 \\
106.226 \\
104.496 \\
105 \cdot 613\end{array}$ & \multirow[b]{2}{*}{0.8665} & \multirow[b]{2}{*}{$7 \cdot 1103$} \\
\hline & & & $105 \cdot 401$ & & \\
\hline \multirow[t]{2}{*}{ Amylbromid } & \multirow[t]{2}{*}{$\begin{array}{l}69.536 \\
71.021 \\
74.801 \\
72.301\end{array}$} & \multirow[t]{2}{*}{$\begin{array}{l}0.53046 \\
0.53767 \\
0.56470 \\
0.54983\end{array}$} & $\begin{array}{l}131 \cdot 086 \\
132 \cdot 091 \\
132 \cdot 461 \\
131 \cdot 497 \\
\end{array}$ & \multirow[b]{2}{*}{1.2161} & \multirow[b]{2}{*}{9.0006} \\
\hline & & & 131.784 & & \\
\hline \multirow[t]{2}{*}{ Chlorbenzol } & \multirow[t]{2}{*}{$\begin{array}{l}143.69 \\
143.18 \\
134.28 \\
126.88\end{array}$} & \multirow[t]{2}{*}{$\begin{array}{l}0.63562 \\
0.63805 \\
0.59667 \\
0.59397\end{array}$} & $\begin{array}{l}226.060 \\
224.406 \\
225.041 \\
223.780 \\
\end{array}$ & \multirow[b]{2}{*}{$1 \cdot 1062$} & \multirow[b]{2}{*}{$12 \cdot 5781$} \\
\hline & & & 224.822 & & \\
\hline \multirow[t]{2}{*}{ Benzylchlorid } & \multirow[t]{2}{*}{$\begin{array}{l}110.40 \\
123.39 \\
105.76\end{array}$} & \multirow[t]{2}{*}{$\begin{array}{l}0.50628 \\
0.55920 \\
0.48371\end{array}$} & $\begin{array}{l}218.052 \\
220.664 \\
218.654\end{array}$ & \multirow[b]{2}{*}{1.1118} & \multirow[b]{2}{*}{13.7128} \\
\hline & & & $219 \cdot 123$ & & \\
\hline \multirow[t]{2}{*}{ Chlortoluol } & \multirow[t]{2}{*}{$\begin{array}{l}137 \cdot 63 \\
143 \cdot 62 \\
136 \cdot 16 \\
134 \cdot 83\end{array}$} & \multirow[t]{2}{*}{$\begin{array}{l}0.66175 \\
0.68277 \\
0.65096 \\
0.64751\end{array}$} & $\begin{array}{l}207 \cdot 986 \\
210 \cdot 347 \\
209 \cdot 160 \\
207 \cdot 744\end{array}$ & \multirow[b]{2}{*}{1.0810} & \multirow[b]{2}{*}{13.4004} \\
\hline & & & 208.809 & & \\
\hline
\end{tabular}

Bei näherer Betrachtung der Zahlen, welche für die elektromagnetische Drehung der substituierten Fettsäuren gefunden wurden, beobachtet man mit dem Eintritt eines Chlor- oder Bromatoms ein Wachsen der Rotation, welches bei den gechlorten Essigsäuren im Mittel 1.3518 und bei den gebromten Essigsäuren 3.2711 für jedes eintretende Chlorund Bromatom beträgt.

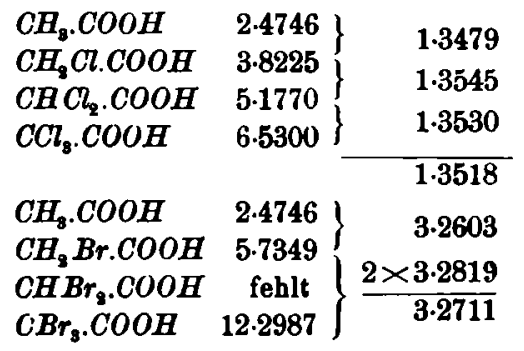




$$
\begin{array}{rr}
\mathrm{CCl}_{3}\left(\mathrm{CH}_{2}\right)_{2} \cdot \mathrm{COOH} & 8.5712 \\
\mathrm{CH}_{2} \cdot\left(\mathrm{CH}_{2}\right)_{2} \cdot \mathrm{COOH} & 4.5465 \\
=4.0267 \\
\mathrm{Cl}=1.3416 .
\end{array}
$$

Die substituierten Essigsäuren sind durch den Austritt von 1, 2 und 3 Wasserstoffatomen und gleichzeitigen Eintritt von ebensoviel Chloratomen gebildet. Legt man die von Perkin ermittelte Drehung der Polarisationsebene durch ein Wasserstoffatom zu Grunde, so berechnet sich daraus die Atomdrehung für Chlor und Brom zu

$$
\begin{array}{rr}
1.352 & 3.271 \\
+0.254 & +0.254 \\
\hline 1.606=C l & -\frac{3.525=B r}{} \text {. }
\end{array}
$$

Die Atomdrehung des Brom beträgt also angenähert das Doppelte der des Chlors. $\mathrm{Zu}$ einem ähnlichen Resultat war $\mathrm{Jahn}$ in seinen Untersuchungen über die Drehung der anorganischen Salze gekommen. Dieselben Atomdrehungen finden sich nun für die genannten Halogene

\begin{tabular}{|c|c|c|c|c|c|}
\hline \multirow{4}{*}{$\begin{array}{l}\text { Amylchlorid } \\
\text { Pentan }\end{array}$} & $\begin{array}{l}7 \cdot 185 \\
5 \cdot 811\end{array}$ & $\begin{array}{l}\text { Amylbromid } \\
\text { Pentan }\end{array}$ & $\begin{array}{l}9.001 \\
5.811\end{array}$ & $\begin{array}{l}\text { Amylenbromid } \\
\text { Pentan }\end{array}$ & $\begin{array}{r}12.669 \\
5.811\end{array}$ \\
\hline & 1.374 & & 3.190 & & 6.858 \\
\hline & 0.254 & & 0.254 & $\boldsymbol{H}_{\mathbf{3}}$ & $=0.508$ \\
\hline & 1.628 & & 3.444 & $B r_{2}$ & $=7.366$ \\
\hline
\end{tabular}
wieder, wenn man die molekularen Drehungen der Chlor- und Bromderivate einiger Kohlenwasserstoffe mit denen der reinen Kohlenwasserstoffe vergleicht. Durch Gegenüberstellung geeigneter Körper, deren Rotation in Tabelle IX angegeben ist, findet man folgende Werte für Chlor und Brom.

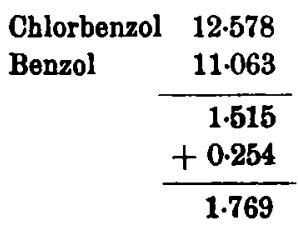

$$
\begin{array}{ll}
\text { Amylbromid } & \mathbf{9 . 0 0 1} \\
\text { Pentan } & \mathbf{5 . 8 1 1} \\
\cline { 2 - 2 } & \mathbf{3 . 1 9 0} \\
& 0.254 \\
\hline & \mathbf{3 . 4 4 4}
\end{array}
$$

Als Mittel für Chlor und Brom werden folgende Zahlen erhalten:

\begin{tabular}{ll}
1.769 & \\
1.628 & 3.444 \\
1.628 & 3.683 \\
\hline $1.675=C l$ & $\frac{3.563}{=B r}$.
\end{tabular}


Ober die elektromagnet. Drehung der Folarisationsebene einiger Sluren etc. 415

Diese Atomdrehungen stimmen befriedigend mit den aus den Säuren abgeleiteten Werten überein, und es scheint die elektromagnetische Rotation der Halogene in den Alkohol- und Säuresubstitutionsprodukten identisch zu sein.

Die Versuche für diese Arbeit wurden auf Veranlassung des Herrn Dr. H. Jahn im zweiten chemischen Universitätslaboratorium während des Wintersemesters 1892/93 ausgefiihrt. Es ist mir eine angenehme Pflicht, auch an dieser Stelle noch Herrn Geheimrat Prof. Dr. Landolt sowie Herrn Dr. Jahn für das rege Interesse, das sie meiner Arbeit entgegengebracht haben, meinen besten Dank auszusprechen.

Berlin, II. chemisches Institut der Universitât, Juli 1893. 